\title{
Giant radio galaxy DA 240 group: content and environment
}

\author{
R. Chen ${ }^{1}$, B. Peng ${ }^{1,2}$, R. G. Strom ${ }^{3,4,5,6}$, J. Wei ${ }^{1}$, and Y. Zhao ${ }^{1}$ \\ 1 National Astronomical Observatories, Chinese Academy of Sciences, Beijing 100012, PR China \\ e-mail: spiritrong@gmail.com \\ 2 Joint Laboratory for Radio Astronomy Technology, Datun Road A20, Beijing 100012, PR China \\ 3 Netherlands Institute for Radio Astronomy (ASTRON), Postbus 2, 7990 AA Dwingeloo, The Netherlands \\ 4 Astronomical Institute "Anton Pannekoek", Faculty of Science, University of Amsterdam, The Netherlands \\ 5 Centre for Astronomy, James Cook University, Townsville QLD 4811, Australia \\ ${ }^{6}$ Department of Physics, National University of Singapore, 2 Science Drive 3, Singapore 117542, Singapore
}

Received 16 April 2010 / Accepted 21 February 2011

\section{ABSTRACT}

\begin{abstract}
Context. There is a group of about twenty galaxies around the giant radio galaxy (GRG) DA 240 which we are studying to investigate the environment of the radio source. We have noted that 11 members are aligned with the radio emission (Peng et al. 2004, A\&A, $415,487)$. The alignment occurs within a small angle similar to the width of the GRG radio lobes, and there may be some physical causal relationship. After finishing observations of the four candidates not included in Paper I, we have completed our study of the brighter members of the sample of galaxies around DA 240.

Aims. We aim to see if more galaxies have similar redshifts to the host galaxy of DA 240, to confirm the galaxy grouping and further study their distribution.

Methods. Spectra of the four candidate galaxies were obtained with the $2.16 \mathrm{~m}$ spectroscopic telescope at XingLong observing station of the NAOC, and the galaxies' positions were established with respect to the radio emission. We have discovered two more members associated with the group of galaxies around DA 240. In addition, the work of other researchers provides one other new member. Results. The number of confirmed galaxies associated with the DA 240 group has increased by almost a quarter to 20 . There are now 14 galaxies (including the host) lying along the major axis of the radio source. We further consider the relationship of the group to adjacent galaxy associations, noting that it may form a binary pair with the slightly closer UGC 3957 group. They both lie on the periphery of the much more substantial cluster, Abell 576.
\end{abstract}

Key words. galaxies: active - galaxies: individual: DA 240 - galaxies: ISM - galaxies: distances and redshifts techniques: spectroscopic - ISM: jets and outflows

\section{Introduction}

Giant radio galaxies (GRGs) are defined as a class with a projected linear size $\geq 1 \mathrm{Mpc}$ (e.g. Klein et al. 1994, for $H_{0}=$ $75 \mathrm{~km} \mathrm{~s}^{-1} \mathrm{Mpc}^{-1}$ ), putting them at the high-end of the linear size distribution. GRGs have been observed at both low and high frequencies to study their morphological character, magnetic field distribution and flux density. It is believed that the lobes of GRGs are moving outwards through the low-density $\left(10^{-5}-10^{-6} \mathrm{~cm}^{-3}\right)$ intergalactic medium (IGM), and their estimated spectral ages are about $10^{7}-10^{8}$ years (Mack et al. 1998; Jamrozy et al. 2008).

DA 240 is a typical GRG with an intermediate value of radio luminosity which lies in the transition region between FR I and II types (Fanaroff \& Riley 1974). It has two lobes with a prominent hotspot in the NE component and a weaker one to the SW. The angular size along the major axis of DA 240 is about 34 arcmin (measured from edge to edge) with a redshift of 0.0356 (Willis et al. 1974). Hence the linear size is $1.4 \mathrm{Mpc}$ (for $H_{0}=71 \mathrm{~km} \mathrm{~s}^{-1} \mathrm{Mpc}^{-1}, \Omega_{\mathrm{m}}=0.27$ and $\left.\Omega_{\Lambda}=0.73\right)$.

In order to investigate the surrounding environment of DA 240, Peng et al. (2004) ${ }^{1}$ obtained optical spectra and found that 16 galaxies had similar redshifts to the host galaxy of DA 240 (redshift of 0.0358 ), and that 10 of the 16 galaxies were distributed along the major axis of the radio emission. Moreover,

\footnotetext{
${ }^{1}$ Hereafter called Paper I.
}

there may be a linear correlation between the relative velocity and projected distance along the source axis.

Here we report on our on-going research on faint galaxies around DA 240. A couple of new members can be added from published results of other research groups. We present our observations and data reduction in Sect. 2, the results and analysis in Sect. 3, a discussion in Sect. 4, and the conclusions in Sect. 5.

\section{Observations and data reduction}

\subsection{Observations}

In Paper I, there were four faint candidates for which we were unable to secure redshifts. These galaxies (listed in Table 1) are the subject of this article. In Table 1, we use the source numbers from Paper I. Columns 2-4 are the Right Ascension, Declination (J2000) and apparent $R$-band magnitude from the USNO-2.0 catalogue.

Following Paper I, we have made spectroscopic observations with the $2.16 \mathrm{~m}$ optical telescope and OMR spectrograph of Xinglong Station, NAOC, with a Tektronix $1024 \times 1024$ CCD as a detector for the first three candidates (Nos. 29, 30, 31). We used the grism of $300 \mathrm{~g} \mathrm{~mm}^{-1}$ with wavelength coverage of 3800 $8000 \AA$. The slit width was $2^{\prime \prime}$ arc and the average spectral resolution was about $9.6 \AA$. Exposures of 3600-7200s were taken on the night of January 29th, 2003. For candidate No. 32, we used 

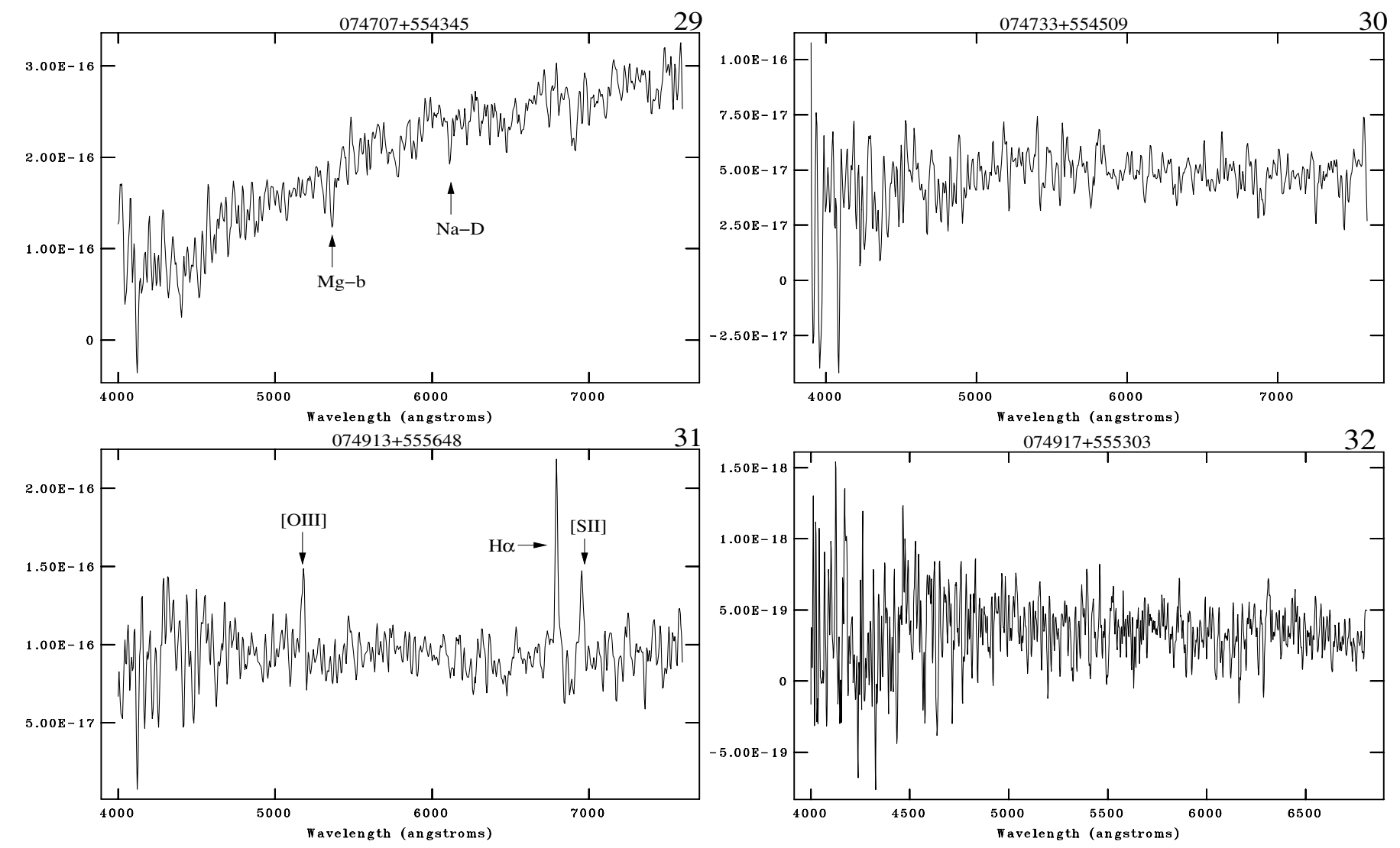

Fig. 1. Spectra of the four galaxies around DA 240, observed with the $2.16 \mathrm{~m}$ optical telescope at Xinglong Station of the NAOC (wavelength not corrected for redshift). The identified lines are marked.

Table 1. Four candidate galaxies from Paper I.

\begin{tabular}{cccc}
\hline \hline No. & $\begin{array}{c}\text { RA (J2000) } \\
\text { h m s }\end{array}$ & $\begin{array}{c}\text { Dec (J2000) } \\
{ }^{\prime}{ }^{\prime}{ }^{\prime}\end{array}$ & $\begin{array}{c}R \\
\text { app. mag }\end{array}$ \\
\hline 29 & 074707.51 & 554345.5 & 15.7 \\
30 & 074733.70 & 554509.0 & 16.8 \\
31 & 074913.49 & 555648.5 & 17.5 \\
32 & 074917.14 & 555303.1 & 17.9 \\
\hline
\end{tabular}

the $\mathrm{BFOSC}^{2}$ spectrograph with a Tektronix $2048 \times 2048 \mathrm{CCD}$ as a detector. We used grism $\mathrm{G} 7$, for which the reciprocal dispersion is $95 \AA / \mathrm{mm}$ with wavelength coverage of $3870-6760 \AA$. The slit width was $1.8^{\prime \prime}$ arc and the average spectral resolution was about $1.4 \AA$. Exposures of $10800 \mathrm{~s}$ were taken on the night of January 6th, 2010. As galaxies are not point sources, the magnitudes given are just indicative values, especially for those with large envelopes. Galaxies with the same indicative magnitude may appear to differ by a lot in an observation. Although we made several attempts, the 32nd candidate of $17.9 \mathrm{mag}$ in the $R$ band was too faint for the OMR spectrograph. It was difficult to align this faint galaxy with the slit and the $\mathrm{S} / \mathrm{N}$ was too low. So we decided to use the BFOSC spectrograph as the detector and took an exposure of three hours. We used helium and argon lamps for the OMR, and Ferrum and argon lamps for the BFOSC to facilitate wavelength calibration. The flux calibration was done by exposures of KPNO standard stars (Massey et al. 1988), such as HD 19445 and G191B2B. The atmospheric extinction was corrected by the mean value for Xinglong. However,

$\overline{2}$ BAO Faint Object Spectrograph and Camera. we did not remove the telluric $\mathrm{O}_{2}$ absorption bands at $6870 \AA$ and $7620 \AA$.

\subsection{Data reduction}

The optical data was reduced with $\mathrm{IRAF}^{3}$ software. After wavelength and flux calibration, we inspected the absorption and emission features of each spectrum. The redshifts were calculated after the identification of several spectral lines, and the average value and its error were finally derived. The reduced spectra are shown in Fig. 1, and their redshifts are listed in Col. 4 of Table 2. Unfortunately, there are no lines in the spectra of Nos. 30 and 32. For this reason no redshift could be determined.

We also made simple classifications for the four objects following the criteria of Veilleux \& Osterbrock (1987). Galaxies without emission lines are classified as normal $(\mathrm{G})$, while objects with $\mathrm{H}_{\alpha}$ emission line but weak forbidden lines of [NII] $6583 \AA$ and [OIII] $5007 \AA$ are classified as HII.

The radio data are from observations made by the Westerbork Synthesis Radio Telescope (WSRT) at a frequency of $608 \mathrm{MHz}$, which consisted of two $12 \mathrm{~h}$ observations with 36 and $72 \mathrm{~m}$ settings of the shortest baseline. The final cleaned radio map was provided by one of us (BP), and some analysis was done using AIPS $^{4}$ software.

\section{Results and analysis}

We have obtained spectra of four additional galaxies around DA 240 which had not been observed for Paper I and are listed

\footnotetext{
3 Image Reduction and Analysis Facility.

4 Astronomical Image Processing System.
} 
Table 2. Summary of the observing results: four galaxies observed with the $2.16 \mathrm{~m}$ optical telescope.

\begin{tabular}{ccccccc}
\hline \hline No. & $\begin{array}{c}\text { RA+Dec name } \\
(\mathrm{J} 2000)\end{array}$ & $\begin{array}{c}\text { Obs. dates } \\
\text { yyyy.mm.dd }\end{array}$ & $\begin{array}{c}\text { Redshift } \\
z\end{array}$ & $\begin{array}{c}\text { Exposure } \\
\text { s }\end{array}$ & Notes & $\mathrm{C}$ \\
\hline 29 & $074707.51+554345.5$ & 2003.01 .29 & $0.0367 \pm 0.0003$ & 3600 & Na-D, Mg-b & $\mathrm{G}$ \\
30 & $074733.70+554509.0$ & 2003.01 .29 & - & 7200 & (no features detected) & $\mathrm{G} ?$ \\
31 & $074913.49+555648.5$ & 2003.01 .29 & $0.0347 \pm 0.0005$ & 3600 & $\mathrm{H}_{\alpha},[\mathrm{SII}],[\mathrm{OIII}]$ & $\mathrm{HII}$ \\
32 & $074917.14+555303.1$ & 2010.01 .06 & - & 10800 & (no features detected) & $\mathrm{G} ?$ \\
\hline
\end{tabular}

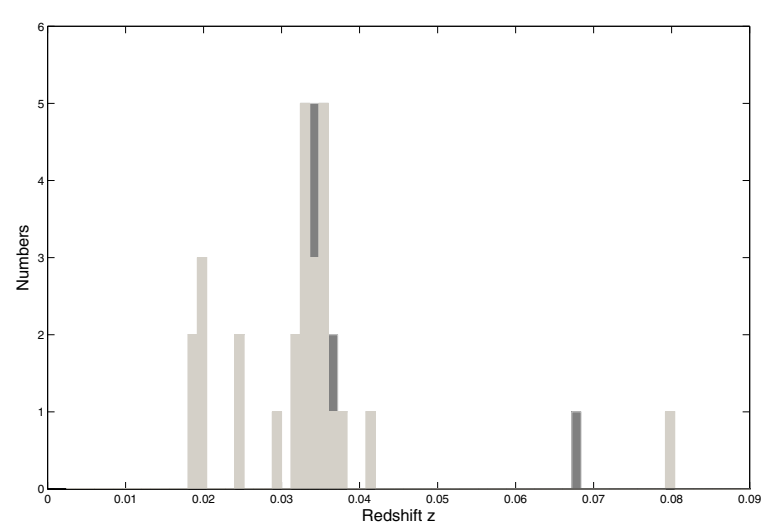

Fig. 2. Histogram of the redshift distribution for the 31 galaxies in the direction of DA 240. The four new redshifts are shown in dark shading, while Nos. 18 (0.1237) and $35(0.174)$ are beyond the region plotted.

in Table 2. Column 1 is the source number and Col. 2 is the coordinate name. Columns 3-5 are the observation dates, redshifts, and exposure times. Column 6 lists the line identifications, and the last column is the classification of galaxy type.

In addition to the four candidates we have observed, the redshifts of other three galaxies recently became available on the NASA/IPAC Extragalactic Database (NED). They are $z=$ 0.034207 (Rines et al. 2000), 0.067296 (Rines et al. 2004) and 0.174 (Stocke et al. 1991). They have been added to our sample, labelled Nos. 33, 34 and 35 respectively, and are included in the discussion below.

\subsection{Redshifts and magnitude}

We have put all the results for the 33 candidates together ${ }^{5}$, and readily find that three new galaxies can be added to the DA 240 main group with $0.032 \leq z \leq 0.038$, as can be seen in a histogram (Fig. 2). The two other galaxies we observed (Table 2) are probably background objects, although their redshifts remain unknown. In any event, the main group now has 20 galaxy members. Figure 3 is the red magnitude distribution of these 20 members, with the host galaxy conspicuously brighter than the rest. This is evidence that the DA 240 host galaxy is the dominant member in terms of stellar mass.

\subsection{Velocity distribution}

We have inspected the velocity distribution of the group members. We take the velocity of galaxy No. 9 (in Paper I we used galaxy No. 6) as our reference point, as it is closest to the median value. From Fig. 4, we find a noticeable peak at about $250 \mathrm{~km} \mathrm{~s}^{-1}$

\footnotetext{
5 The redshifts and coordinates of all 33 galaxies are listed in Appendix Table A.1.
}

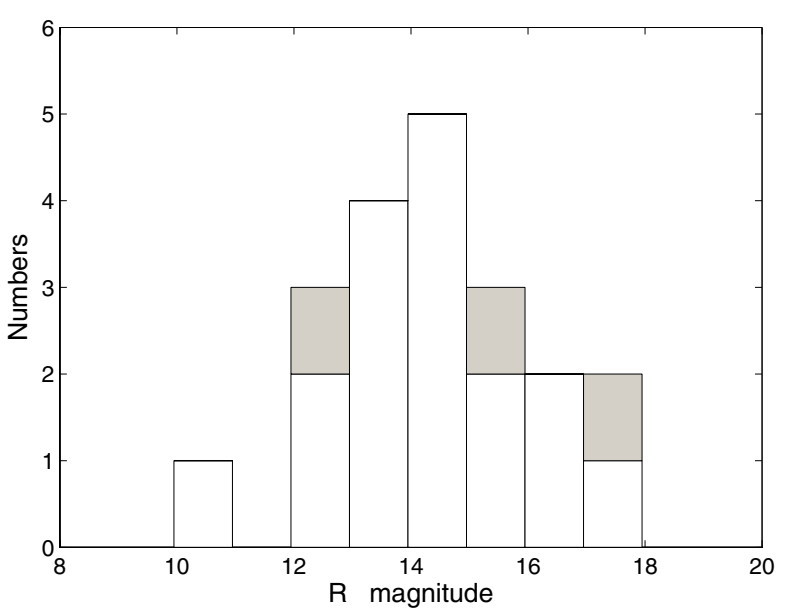

Fig. 3. Magnitude distribution of the 20 galaxies in the main group. The additional three members are shown with a darker shade.

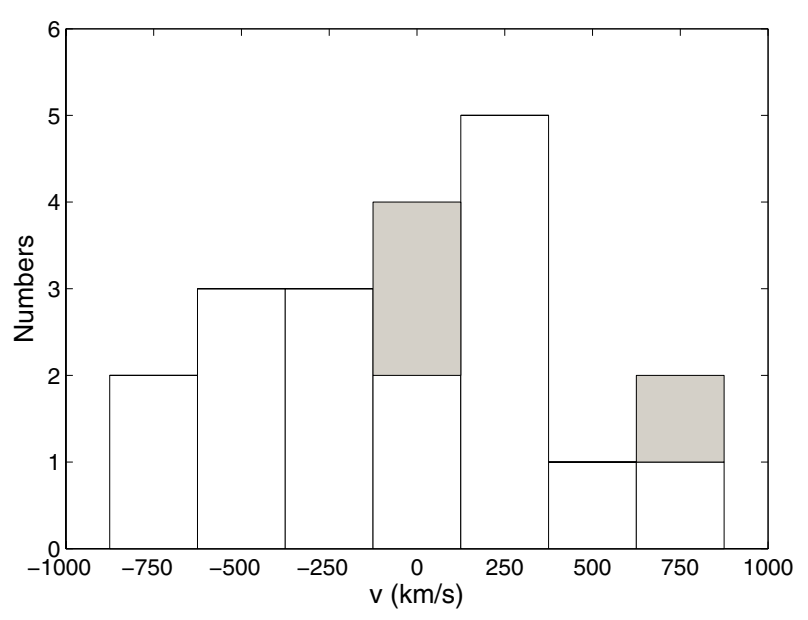

Fig. 4. Velocity distribution of the 20 group members. The additional three are shown with dark shading.

in the velocity distribution. Comparing it with a Gaussian distribution of the same mean $v$ and $\sigma$, a $\chi^{2}$ test indicates that the probability of the velocities being normally distributed is about $32 \%$ (15\% in Paper I): the distribution does not differ significantly from a Gaussian.

\subsection{Locations and dynamic lifetime}

The locations of the 20 main group galaxies are illustrated together with the $49 \mathrm{~cm}$ radio brightness distribution in Fig. 5. The three additional galaxies are displayed with different symbols. Two are in one lobe while one is in the other: galaxy Nos. 31, 33 are in the NE lobe while galaxy No. 29 lies in the SW 


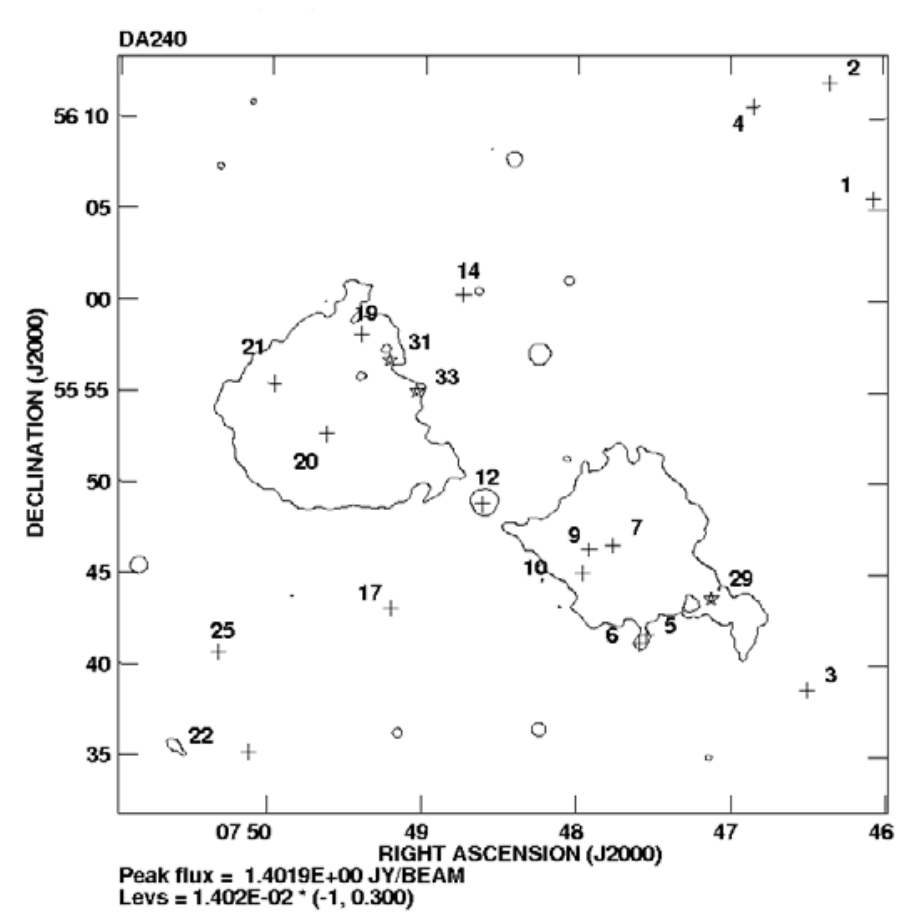

Fig. 5. DA 240 single-contour map (showing the radio extent) obtained with the WSRT at $49 \mathrm{~cm}$, with 20 optical galaxies at redshifts $0.032 \leq$ $z \leq 0.038$. The previous 17 members are plotted with crosses while the three additions (Nos. 29, 31 and 33) are with stars.

one. So, there are six galaxies (including galaxy No. 14) around the NE lobe and seven around the SW lobe (including No. 3). Another striking feature of the galaxy distribution is the absence of any close companions to VV 9-13-57 (the DA 240 host). The nearest group member is galaxy No. 9, at a projected distance of some $250 \mathrm{kpc}$ from VV 9-13-57. We will return to this point in Sect. 4 below.

We can compute the dynamic lifetime of each galaxy group to compare it with the radiation age of the respective lobe as was done in Paper I. Since one can only measure the line-of-sight velocity, we have corrected the velocity dispersions to account for this. For the eastern group, the dispersion is, $\sigma=478 \mathrm{~km} \mathrm{~s}^{-1}$, and $766 \mathrm{~km} \mathrm{~s}^{-1}$ for the western group. Considering the lobe radii are both about $400 \mathrm{kpc}$, it is straightforward to derive dynamic lifetimes of $8 \times 10^{8} \mathrm{yr}$ for eastern group and $5 \times 10^{8} \mathrm{yr}$ for western group. We quoted a value for the radiation age in Paper I, which was $t \leq 10^{7} \mathrm{yr}$. Hence the kinematic lifetime of the galaxy groups is comfortably larger than the radiation age of the radio lobes.

\subsection{Monte-Carlo simulation}

There are now 13 galaxies (excluding No. 14) lying along the major axis as defined by the radio core and hotspots of DA 240. In order to test whether the alignment of the 13 members is significant, a Monte-Carlo simulation has been performed. The lobes are constrained by two lines passing through galaxy No. 12 (VV 9-13-57), $19^{\circ}$ on each side of the major axis. With 13 galaxies in this defined area, we computed the probability of 13 or more appearing there by chance. Locations of the 20 members were randomly generated, and the process was repeated $10^{7}$ times. This experiment showed that the configuration observed would happen by chance at a rate of $7 \times 10^{-6}$, compared with the value of $3 \times 10^{-4}$ in Paper I, where the number of group members was 17 and only 10 appeared in the same constrained area. The three additional galaxies strengthen the case that the alignment is physically significant.

\subsection{Relative distance and velocity}

We have also looked into the kinematics of the 14 galaxies lying in or near the radio lobes. After projecting locations of the galaxies onto the radio axis, we have computed their projected distance from galaxy No. 12. In this way we have generated an updated version (not shown) of Fig. 7 which appeared in Paper I. We find that there is still a correlation between $\Delta v$ and $D$, although the correlation coefficient has decreased to -0.67 from the -0.74 of Paper I. And after a least-squares fit to those points, the gradient is -0.391 (up from -0.425 in Paper I), indicating $\Delta v / D=391 \mathrm{~km} \mathrm{~s}^{-1} \mathrm{Mpc}^{-1}$. We can also compute the enclosed mass for the case of galaxies seen in an edge-on ring, orbiting the center. For $d / 2=r \simeq 1 \mathrm{Mpc}$ and $v_{\text {orb }} \simeq 391 \mathrm{~km} \mathrm{~s}^{-1}$, the mass is $M=r v_{\text {orb }}^{2} / \mathrm{G}=3.5 \times 10^{13} M_{\odot}$.

\section{Discussion}

We begin by considering the larger-scale environment in which the DA 240 group is embedded. As already noted in Paper I, DA 240 lies on the western edge of the Zwicky cluster Zw 0756.1+5616 (Zwicky \& Herzog 1966). Almost $4^{\circ}$ to the west lies another large cluster, Abell 576, at a somewhat greater distance $(z \simeq 0.039)$ than DA 240 . Optical information on A576 can be found in the catalogue of Hudson et al. (2001). After the publication of Paper I, the NOAO Fundamental Plane Survey appeared (Smith et al. 2004). It includes redshift information on the UGC 3957 galaxy group. UGC 3957 lies between DA 240 and A576, at a redshift of $z=0.034$. It consists of more galaxies (over 50 spectroscopically confirmed and listed in NED) than the DA 240 group, but has a similar diameter and only a slightly smaller redshift. The two may form a binary system, a not uncommon phenomenon (Karachentsev \& Kopylov 1981; Karachentsev \& Shcherbanovskii 1978; and Ulmer et al. 1985). However, they appear to be well-separated both spatially (Fig. 6) and in distance ( $c z$ differs by $170 \mathrm{~km} \mathrm{~s}^{-1}$, or $2.3 \mathrm{Mpc}$ ), and may be too remote from one another (total separation of $\sim 3.4 \mathrm{Mpc}$ if the velocity difference is purely due to Hubble expansion, although this is not necessarily the case if the two groups are interacting dynamically) to be considered a true binary.

In Fig. 6, we show the locations of all galaxies within a radius of $8^{\circ}$ from the DA 240 host and in the redshift range of the group, $0.032 \leq z \leq 0.038$. The two central concentrations are the UGC 3957 and DA 240 galaxy groups. To the west and slightly north, we have A576. Its central condensation is clearly visible, despite the fact that the $z \leq 0.038$ upper limit means that only about half of the galaxies in the cluster are shown in this figure. Most of the galaxies to the east belong to $\mathrm{Zw} 0756.1+5616$. The limit of the SDSS region runs through the center of the figure near RA $=8^{\mathrm{h}}$; the spectra of many of the galaxies east of this line come from the SDSS. Figure 7 shows the distribution in redshift for galaxies in four regions of sky centered on the two groups, and the Abell and Zwicky clusters. These histograms clearly show that A576 has a somewhat higher average redshift than the groups and $\mathrm{Zw} 0756.1+5616$, though there is significant overlap in the lower- $z$ wing. The DA 240 and UGC 3957 groups, and the Zwicky cluster, overlap considerably in redshift, though they are spatially distinct. 


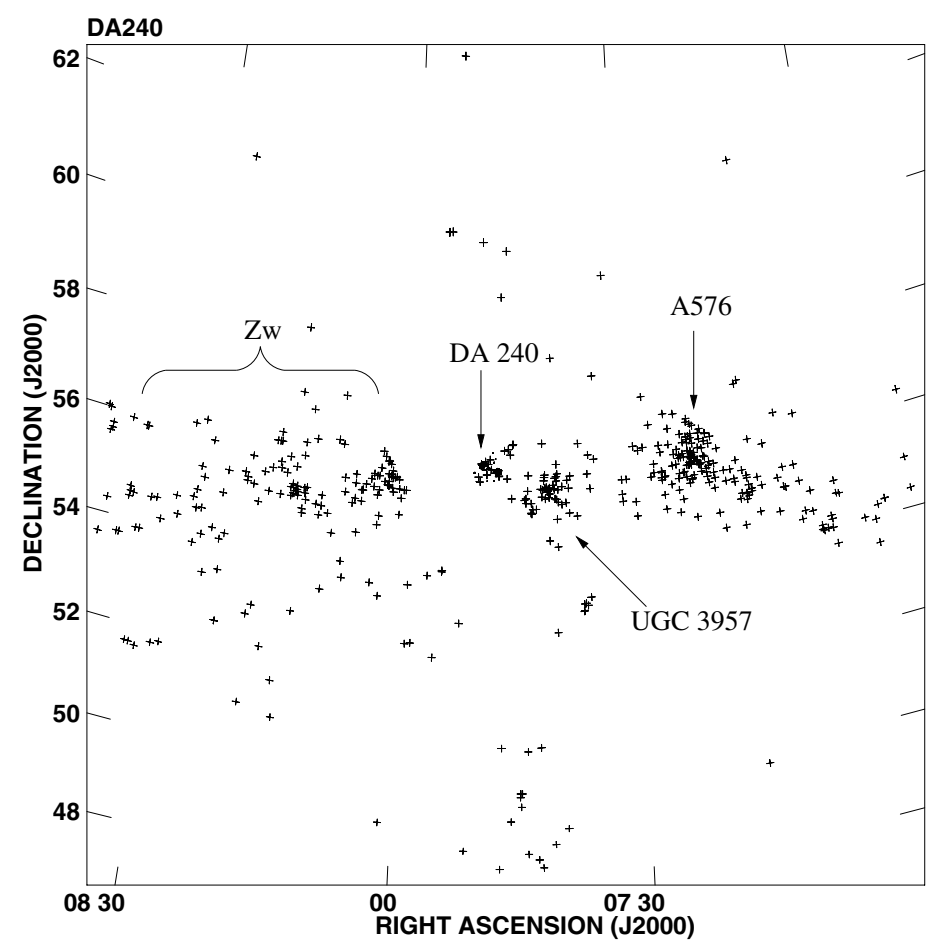

Fig. 6. Locations of all galaxies within a radius of $8^{\circ}$ from the DA 240 host and in the redshift range of $0.032 \leq z \leq 0.038$. Rough locations of the four clusters/groups are indicated, where $\mathrm{Zw}$ stands for the Zwicky cluster Zw $0756.1+5616$.

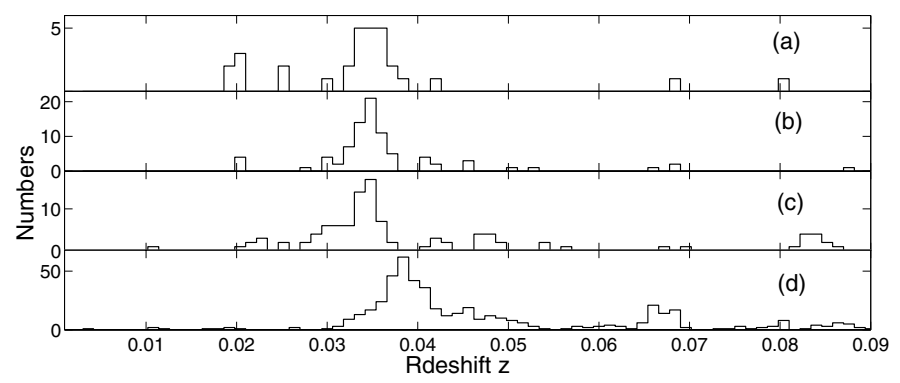

Fig. 7. Redshfit distributions of four groups: a) DA 240; b) UGC 3957; c) Zw 0756.1+5616; d) Abell 576 .

In Fig. 8 we show, for all galaxies in the same region of space as Fig. 6, but over the redshift range $0.02 \leq z \leq 0.05$, a plot of RA vs. $c z$. A576 is clearly seen near RA $=7.4^{\mathrm{h}}$, $c z=11500 \mathrm{~km} \mathrm{~s}^{-1}$. There appears to be a filament from A576, going to higher RA and higher $v$. From an examination of the RA, Dec, velocity cube (not shown), we conclude that this feature has a nearly constant Dec. Zw $0756.1+5616$ is not as concentrated as A576, and most of its members fall in the range, $8^{\mathrm{h}} \leq \mathrm{RA} \leq 8.6^{\mathrm{h}}$. The DA 240 and UGC 3957 groups fall in a gap between the Abell and Zwicky clusters. Although there are clearly fewer galaxies east and west of the two groups, one might wonder whether the gaps are related to the border of the SDSS survey region. We would argue that this should equally affect the higher-velocity filament (Fig. 8), yet it is almost continuous (there is a slight deficiency of galaxies near RA $=7.75^{\mathrm{h}}$ ), without the same gaps that isolate the DA 240 and UGC 3957 groups. We conclude that although there are other associations in the (both spatial and velocity) neighborhood, the two small groups form a separate structure, possibly a binary in which the UGC 3957 group dominates. Binary galaxy clusters may be in

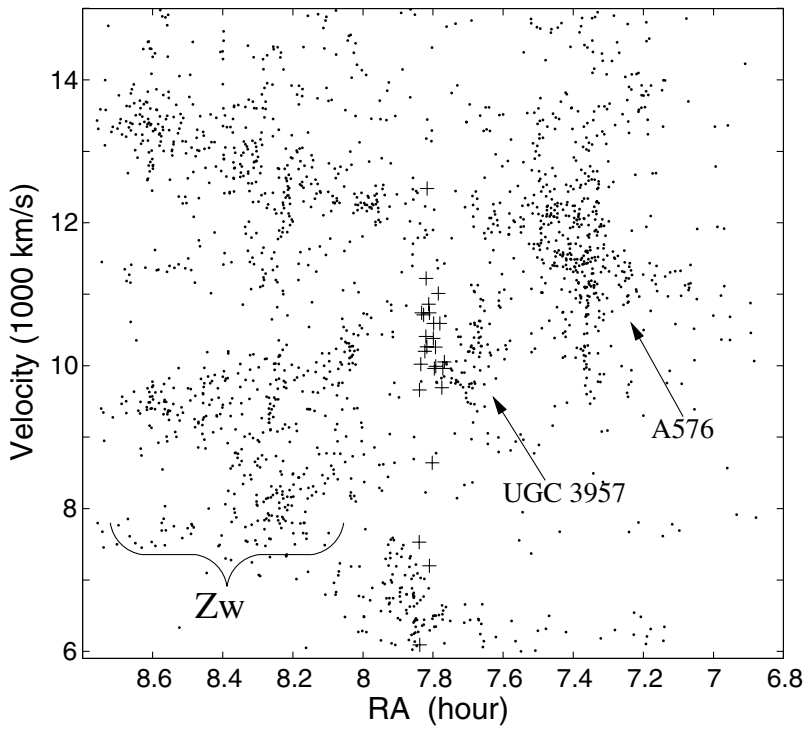

Fig. 8. Velocity vs. Right Accession. Galaxies in the DA 240 sample are marked with crosses. The other group and two clusters are indicated using the same symbols as in Fig. 6.

the process of merging and this could trigger activity in their constituent galaxy members (Hwang \& Lee 2009). As we have noted before (Peng et al. 2004), there is an excess of active galaxies in the DA 240 group.

What is the environment of other GRGs? Recent work on two southern sources can help put things in a broader context. MSH 05-22 and MRC B0319-454 are somewhat larger than DA 240; morphologically, they are fairly typical of GRGs, with collimated lobes which decrease in brightness with increasing distance from the head. Jet structures are observed in both sources, and the relative lobe length is quite unequal, which is not the case for DA 240. In a study of MSH 05-22, Subrahmanyan et al. (2008) find that the source is located 1$2 \mathrm{Mpc}$ south of the center of the group of which the host is a member. This group is on the edge of a higher-density concentration in the form of a sheet or filament. The southern radio lobe is some $60 \%$ longer than its northern counterpart; the latter is also displaced laterally with respect to the host location. A possible explanation for the differences in lobe structure is a wind flowing out from the galaxy concentration to the northeast, and both displacing and retarding the northern radio lobe.

MRC B0319-454 shares some of the characteristics of MSH 05-22, but there are also differences (Safouris et al. 2009). Both are associated with small galaxy groups on the periphery of larger conglomerations. In MRC B0319-454, the more distant lobe leading edge, which ends in a bright hotspot, is over twice as far from the host as its counterpart to the northeast. The host has half a dozen companions which form a small group around the (shorter) northeastern lobe, while most of the galaxies of similar redshift are scattered to the southwest and west. The southwestern component trails off from the hotspot almost perpendicular to the source major axis. A plausible explanation for this behavior can be found in buoyancy of the jet backflow (Safouris et al. 2009). DA 240 is different from these two sources in several respects. In terms of the radio morphology, its lobes are much more symmetrical, both contain hotspots (though of very different intensity) and the low surface brightness morphology is roughly circular (though the western lobe 
has a "tongue" of emission leading its hotspot). The galaxies in the DA 240 group are rather symmetric about the host, with a majority lying along the radio source axis. One thing all three radio sources share is that they are associated with small groups on the edge of much larger galaxy clusters.

Let us now try to put some of our results into the context of what is more generally known about medium-sized groups of galaxies, of which both the UGC 3957 and DA 240 groups are evident examples. Tully (1987) has collected a sample of well over 100 groups and other associations based upon surveys, for $c z<3000 \mathrm{~km} \mathrm{~s}^{-1}$. He has taken great care in defining what constitutes an association in his sample. The most relevant subset are the 49 groups with five or more members, which Tully has extensively investigated. He presents information on the collective fundamental physical properties of his sample, which can be used as a benchmark for the DA 240 group. He finds, for example, that the distribution of galaxy velocities about the mean is symmetrical, just as we see (Fig. 4). The mean velocity dispersion in his sample, however, is $100 \mathrm{~km} \mathrm{~s}^{-1}$, considerably lower than the $434 \mathrm{~km} \mathrm{~s}^{-1}$ of the DA 240 group, though similar to that of UGC 3957.

Various studies have shown that there are correlations between the velocity dispersions of clusters of galaxies and the temperature and X-ray luminosity of their hot gas. Mulchaey \& Zabludoff (1998), for example, show that the X-ray temperature and velocity dispersion of groups are correlated and form an extension of the correlation found for clusters. A similar relationship is found between velocity dispersion and X-ray luminosity, with again groups falling on an extension of the cluster correlation. Attempts to detect X-ray emission from DA 240 and its surroundings have been only partially successful. Miller et al. (1999) used the ROSAT All Sky Survey data to set a limit to the DA $240 \mathrm{X}$-ray luminosity of about $10^{42} \mathrm{erg} \mathrm{s}^{-1}$. Evans et al. (2008) used XMM-Newton to detect X-rays from VV 9-13-57 at a level of $5.5 \times 10^{40} \mathrm{erg} \mathrm{s}^{-1}$. This unresolved emission originates, they conclude, in the parsec-scale jet in the galaxy's nucleus. They also present an X-ray map of the entire GRG showing no trace of extended emission, confirming the Miller et al. (1999) upper limit.

It is a bit surprising to find no X-ray emission from the center of the DA 240 group, given its substantial velocity dispersion. According to the correlations found by Mulchaey \& Zabludoff (1998), the group might be expected to have an X-ray luminosity of $10^{43} \mathrm{erg} \mathrm{s}^{-1}$ with a gas temperature slightly in excess of $1 \mathrm{keV}$, although there is significant scatter in the data. There are at least two other unusual facts about the DA 240 group. The galaxies in poor groups are almost always concentrated toward the center (see Beers et al. 1995, for numerous examples; this is, of course, also true of clusters). In the case of the DA 240 group, there is a conspicuous absence of galaxies in the vicinity of VV 9-1357. As noted above, its nearest neighbor is No. 9, at a projected distance of $250 \mathrm{kpc}$. The fact that VV 9-13-57 lies at the geometrical center of the group is as expected (Beers et al. 1995), but unusually, kinematically, the galaxy is offset by $+370 \mathrm{~km} \mathrm{~s}^{-1}$ from the group average, $10360 \mathrm{~km} \mathrm{~s}^{-1}$. Beers et al. (1995) find that in $80 \%$ of the poor clusters they studied, the central galaxies show relatively small velocity offsets.

We have, at the moment, no explanation for the lack of a hot, substantially dense gaseous component, nor for the absence of galaxies near the center of the group. Perhaps it is somehow related to the binary nature of the group, if it is still in the process of merging. We note that both a lower ambient density and the absence of nearby companions are both consistent with the presence of a GRG, and one with broad components which have

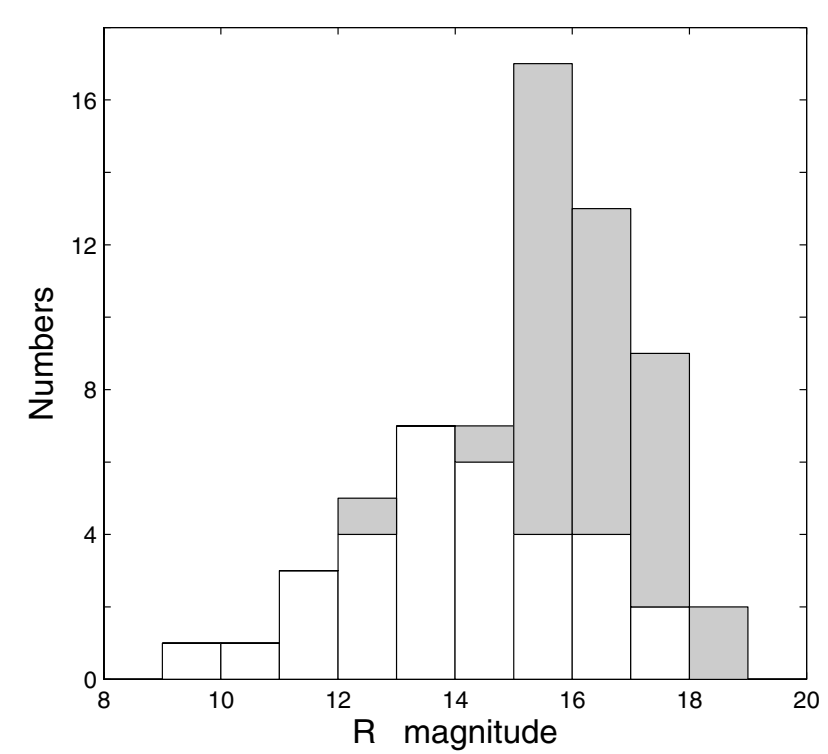

Fig. 9. A histogram showing the R-magnitudes of the galaxies we have studied (Table A.1) traced with a solid line, and in shading the magnitudes of the remaining USNO galaxies.

expanded laterally almost as far as they have moved away from the host.

\section{Conclusions}

Apart from the two faint members (Nos. 30 and 32) we have essentially completed our spectral investigation of galaxies within $30^{\prime}$ arc of DA 240 . We have redshifts for 33 galaxies on our original list. There is, to be sure, roughly an equal number of additional galaxies in the same region of sky from the USNO catalogue, but they are fainter than most of the galaxies in our original sample. The $R$-band magnitudes for these uninvestigated objects are compared with those from the sample of 33 in Fig. 9, showing that they are mainly on the faint side of the distribution. There remains one fairly bright $\left(R=12^{\mathrm{m}}\right)$ galaxy, $\mathrm{J} 075150+554119$ from the USNO A2 list; it is at the extreme SE end of our search zone.

Apart from $\mathrm{J} 075150+554119$, our investigation of the DA 240 group is practically complete for all galaxies with $R<$ $15^{\mathrm{m}}$ within $1.2 \mathrm{Mpc}$ of the radio source host. Since there may be fainter members of the group, our conclusions apply to the brighter, dominant galaxies.

In this paper, we have added three candidates to the confirmed members of the DA 240 group. All three of these galaxies lie near the lobes, increasing the number of galaxies lying along the radio major axis from 11 to 14 . This strengthens the case for alignment not having arisen by chance, as shown by our MonteCarlo simulation. The kinematic timescale for group members which cluster in or near the lobes is also clearly larger than the lobes' radiation ages. Furthermore, as found in Paper I, there is a correlation between the relative projected distance and velocity.

Our work confirms the results of Paper I, though they still remain unexplained. We have also looked into the larger-scale environment around the DA 240 group, and find that it has a "sister" group of similar size, and that the two are not far from a substantial Abell cluster. The likelihood that the two groups may still be merging could affect activity of the individual galaxies. The absence of galaxies and dense gas within $250 \mathrm{kpc}$ of the 
host galaxy helps explain the huge size of the radio lobes: there is hardly anything to impede their expansion. To continue this investigation of the environments of GRGs, we are extending the study to other nearby sources.

Acknowledgements. We are grateful to the referee, Dr. D. J. Saikia, for a number of suggestions which have considerably improved the paper. This work was partially supported by the Open Project Program of the Key Laboratory of Optical Astronomy, NAOC, CAS. The Westerbork Synthesis Radio Telescope is operated by The Netherlands Institute for Radio Astronomy (ASTRON), with financial support from The Netherlands Organization for Scientific Research (NWO). We thank the Chinese and Royal Netherlands Academies of Sciences (CAS and KNAW) for financial support under a bilateral exchange programme which has facilitated travel by B.P. and R.G.S., R.G.S. is also grateful to the CAS for the award of a Visiting Professorship for Senior International Scientists. We also thank Dr. C. Jin and Dr. J. Wang for their help with the data reduction, and Dr. $\mathrm{H}$. Wu for providing extra telescope time when weather curtailed an observing run. This research has made use of the NASA/ADS Abstract Service, and of NED which is operated by the Jet Propulsion Laboratory, Caltech, under contract with the National Aeronautics and Space Administration. We are grateful for the use of the USNO-A2.0 catalogue.

\section{References}

Beers, T. C., Kriessler, J. R., Bird, C. M., \& Huchra, J. P. 1995, AJ, 109, 874 Evans, D. A., Hardcastle, M. J., Lee, J. C., et al. 2008, ApJ, 688, 844

Fanaroff, B. L., \& Riley, J. M. 1974, MNRAS, 167, L31

Hudson, M. J., Lucey, J. R., Smith, R. J., Schlegel, D. J., \& Davies, R. L. 2001, MNRAS, 327, 265

Hwang, H. S., \& Lee, M. G. 2009, MNRAS, 397, 2111

Jamrozy, M., Konar, C., Machalski, J., \& Saikia, D. J. 2008, MNRAS, 385, 1286

Karachentsev, I. D., \& Kopylov, A. I. 1981, Sov. Astr. L., 7, 285

Karachentsev, I. D., \& Shcherbanovskii, A. L. 1978, Sov. Astr., 22, 257

Klein, U., Mack, K.-H., Strom, R. G., Wielebinski, R., \& Achatz, U. 1994, A\&A, 283, 729

Mulchaey, J. S., \& Zabludoff, A. I. 1998, ApJ, 496, 73

Mack, K. H., Klein, U., O’ Dea, C. P., Willis, A. G., \& Saripalli, L. 1998, A\&A, 329,431

Massey, P., Strobel, K., Barnes, J. V., \& Anderson, E. 1988, ApJ, 328, 315

Miller, N. A., Owen, F. N., Burns, J. O., Ledlow, M. J., \& Voges, W. 1999, AJ, 118,1988

Lang, K. R. 1999, Astrophysical Formulae, 2 vols (Berlin: Springer-Verlag)

Peng, B., Strom, R. G., Wei, J., \& Zhao, Y. H. 2004, A\&A, 415, 487

Rines, K., Geller, M. J., Diaferio, A., Mohr, J. J., \& Wegner G. A. 2000, AJ, 120, 2338

Rines, K., Geller, M. J., Diaferio, A., Kurtz, M. J., \& Jarrett, T. H. 2004, AJ, 128, 1078

Safouris, V., Subrahmanyan, R., Bicknell, G. V., \& Saripalli, L. 2009, MNRAS, 393,2

Smith, R. J., Hudson, M. J., Nelan, J. E., et al. 2004, AJ, 128, 1558

Stocke, J. T., Morris, S. L., Gioia, I. M., et al. 1991, ApJS, 76, 813

Subrahmanyan, R., Saripalli, L., Safouris, V., \& Hunstead, R. W. 2008, ApJ, 677, 63

Tully, R. B. 1987, ApJ, 321, 280
Ulmer, M. P., Cruddace, R. G., \& Kowalski, M. P. 1985, ApJ, 290, 551 Veilleux, S., \& Osterbrock, D. E. 1987, ApJS, 63, 295

Willis, A. G., Strom, R. G., \& Wilson, A. S. 1974, Nature, 250, 625

Zwicky, F., \& Herzog, E. 1966, Catalogue of Galaxies and Clusters of Galaxies (Pasadena: California Institute of Technology)

\section{Appendix A}

An overview of all the galaxies in our DA 240 sample is provided in Table A.1.

Table A.1. Thirty-three galaxies in the DA 240 sample.

\begin{tabular}{lcccc}
\hline \hline No. & RA (J2000) & Dec $(\mathrm{J} 2000)$ & $R$ & $z$ \\
& $\mathrm{~h} \mathrm{~m} \mathrm{~s}$ & ${ }^{\prime} \mathrm{s}$ & app. mag & \\
\hline 1 & 074603.94 & 560536.2 & 13.1 & 0.0335 \\
2 & 074620.83 & 561157.8 & 13.2 & 0.0332 \\
3 & 074630.26 & 553844.2 & 14.6 & 0.0323 \\
4 & 074651.07 & 561042.1 & 14.2 & 0.0353 \\
5 & 074732.90 & 554148.2 & 16.5 & 0.0333 \\
6 & 074735.52 & 554118.5 & 12.6 & 0.0342 \\
7 & 074745.94 & 554641.4 & 17.2 & 0.0332 \\
8 & 074751.26 & 554706.7 & 15.7 & 0.0800 \\
9 & 074755.12 & 554628.3 & 13.9 & 0.0353 \\
10 & 074757.75 & 554510.1 & 15.0 & 0.0346 \\
11 & 074808.29 & 554815.5 & 14.1 & 0.0288 \\
12 & 074836.87 & 554858.3 & 10.7 & 0.0358 \\
13 & 074836.87 & 554858.3 & 16.4 & 0.024 \\
14 & 074844.76 & 560035.5 & 16.8 & 0.0362 \\
15 & 074901.27 & 552532.0 & 13.9 & 0.0416 \\
16 & 074909.84 & 553616.8 & 11.4 & 0.0183 \\
17 & 074912.28 & 554312.2 & 14.2 & 0.0374 \\
18 & 074912.62 & 555233.6 & 14.7 & 0.1237 \\
19 & 074924.56 & 555813.4 & 14.1 & 0.0340 \\
20 & 074937.90 & 555244.4 & 14.3 & 0.0357 \\
21 & 074958.51 & 555528.0 & 12.7 & 0.0358 \\
22 & 075007.12 & 553514.5 & 13.2 & 0.0334 \\
23 & 075008.40 & 552303.0 & 9.5 & 0.0193 \\
24 & 075016.36 & 552429.9 & 11.0 & 0.0203 \\
25 & 075019.34 & 554041.5 & 15.0 & 0.0322 \\
26 & 075021.33 & 555401.4 & 11.7 & 0.0251 \\
27 & 075028.99 & 552901.8 & 13.0 & 0.0190 \\
28 & 075053.10 & 552758.1 & 12.8 & 0.0196 \\
29 & 074707.51 & 554345.5 & 15.7 & 0.0367 \\
31 & 074913.49 & 555648.5 & 17.5 & 0.0347 \\
33 & 074902.7 & 555507 & 12.2 & 0.034207 \\
34 & 074527.5 & 554728 & 13.9 & 0.067296 \\
35 & 074909.3 & 553755 & 16.6 & 0.1740 \\
\hline & & & &
\end{tabular}

